CP3-ORIGINS-2010-8

\title{
ON THE EXISTENCE OF HARMONIC MORPHISMS FROM THREE-DIMENSIONAL LIE GROUPS
}

\author{
SIGMUNDUR GUDMUNDSSON AND MARTIN SVENSSON \\ To Professor John $\mathbb{C}$ Wood on his sixtieth birthday.
}

\begin{abstract}
In this paper we classify those three-dimensional Riemannian Lie groups which admit harmonic morphisms to surfaces.
\end{abstract}

\section{INTRODUCTION}

A harmonic morphism between two Riemannian manifolds is a map with the property that its composition with any local harmonic function on the target manifold is a local harmonic function on the domain. These maps can be seen as an extension of conformal mappings between Riemann surfaces. Harmonic morphisms were introduced by C. G. J. Jacobi [8], but the first characterization of these in the context of Riemannian manifolds was made by B. Fuglede and T. Ishihara [4, 7]. Not surprisingly, harmonic morphisms must solve a non-linear, overdetermined system of partial differential equations. Therefore, there is no general existence theory; in fact, there are examples of Riemannian manifolds which do not allow any global, non-constant harmonic morphism between them [9].

With this in mind, it is natural to consider low-dimensional situations when attempting to classify harmonic morphisms. A submersive harmonic morphism gives rise to a conformal foliation of its domain, and when the target manifold is a surface, the leaves of this foliation are minimal submanifolds. Hence any submersive harmonic morphism from a 3-manifold to a surface gives rise to a conformal foliation by geodesics of the domain. In a careful study of this situation, P. Baird and J. C. Wood proved that the Ricci curvature must be conformal on the distribution orthogonal to the leaves. It follows from this that there can be at most two distinct conformal foliations by geodesics of a 3-manifold with non-constant sectional curvature, and a similar result is then true for the number of possible harmonic morphisms. For precise statements of these results, we refer the reader to Section 10.6 of [3] or Theorem 4.1 below. By using this, Baird and Wood showed that the 3-dimensional Thurston geometry Sol (see Section 3 below) does not allow any non-constant harmonic morphisms to a surface, not even locally.

We will here apply the results of Baird and Wood on conformal foliations by geodesics to 3-dimensional Lie groups. We give a complete classification

2000 Mathematics Subject Classification. 58E20, 53C43, 53C12.

Key words and phrases. harmonic morphisms, minimal submanifolds, Lie groups. 
of those 3-dimensional Riemannian Lie groups admitting harmonic morphisms to surfaces. In particular, we show that Sol fits into a continuous family of Lie groups, none of which admits any non-constant local harmonic morphisms to surfaces.

For the general theory of harmonic morphisms, we refer to the exhaustive book [3] or the on-line bibliography of papers [5].

\section{HARMONIC MORPHISMS AND MINIMAL CONFORMAL FOLIATIONS}

Let $M$ and $N$ be two manifolds of dimensions $m$ and $n$, respectively. A Riemannian metric $g$ on $M$ gives rise to the notion of a Laplacian on $(M, g)$ and real-valued harmonic functions $f:(M, g) \rightarrow \mathbb{R}$. This can be generalized to the concept of harmonic maps $\phi:(M, g) \rightarrow(N, h)$ between Riemannian manifolds, which are solutions to a semi-linear system of partial differential equations, see [3].

Definition 2.1. A map $\phi:(M, g) \rightarrow(N, h)$ between Riemannian manifolds is called a harmonic morphism if, for any harmonic function $f: U \rightarrow \mathbb{R}$ defined on an open subset $U$ of $N$ with $\phi^{-1}(U)$ non-empty, $f \circ \phi: \phi^{-1}(U) \rightarrow$ $\mathbb{R}$ is a harmonic function.

The following characterization of harmonic morphisms between Riemannian manifolds is due to Fuglede and Ishihara. For the definition of horizontal (weak) conformality we refer to $[3]$.

Theorem 2.2. [4, 7] A map $\phi:(M, g) \rightarrow(N, h)$ between Riemannian manifolds is a harmonic morphism if and only if it is a horizontally (weakly) conformal harmonic map.

The next result gives the theory of harmonic morphisms a strong geometric flavour. It also shows that the case when the codomain is a surface is particularly interesting.

Theorem 2.3. [1] Let $\phi:\left(M^{m}, g\right) \rightarrow\left(N^{n}, h\right)$ be a horizontally (weakly) conformal map between Riemannian manifolds. If

i. $n=2$, then $\phi$ is harmonic if and only if $\phi$ has minimal fibres at regular points;

ii. $n \geq 3$, then two of the following conditions imply the other:

(a) $\phi$ is a harmonic map,

(b) $\phi$ has minimal fibres at regular points,

(c) $\phi$ is horizontally homothetic.

In particular, the conditions characterizing harmonic morphisms into a surface $N^{2}$ only depend on the conformal structure of $N^{2}$.

\section{The 3-Dimensional LiE GRoups}

At the end of the 19th century, L. Bianchi classified the 3-dimensional real Lie algebras. They fall into nine disjoint types I-IX. Each contains a single isomorphy class except types VI and VII which are continuous families of 
different classes. For later reference we list below Bianchi's classification and notation for the corresponding simply connected Lie groups. We also equip these Lie groups with the left-invariant metric for which the given basis $\{X, Y, Z\}$ of each Lie algebra is orthonormal at the identity.

Example 3.1 (Type I). The Abelian Lie algebra $\mathbb{R}^{3}$; the corresponding simply connected Lie group is of course the Abelian group $\mathbb{R}^{3}$ which we equip with the standard flat metric.

Example 3.2 (II). The Lie algebra $\mathfrak{n}_{3}$ with a basis $X, Y, Z$ satisfying

$$
[X, Y]=Z \text {. }
$$

The corresponding simply connected Lie group is the nilpotent Heisenberg group $\mathrm{Nil}^{3}$.

Example 3.3 (III). The Lie algebra $\mathfrak{h}^{2} \oplus \mathbb{R}=\operatorname{span}\{X, Y, Z\}$, where $\mathfrak{h}^{2}$ is the two-dimensional Lie algebra with basis $X, Y$ satisfying

$$
[Y, X]=X \text {. }
$$

The corresponding simply connected Lie group is denoted by $H^{2} \times \mathbb{R}$. Here $H^{2}$ is the standard hyperbolic plane.

Example 3.4 (IV). The Lie algebra $\mathfrak{g}_{4}$ with a basis $X, Y, Z$ satisfying

$$
[Z, X]=X, \quad[Z, Y]=X+Y \text {. }
$$

The corresponding simply connected Lie group is denoted by $G_{4}$.

Example 3.5 (V). The Lie algebra $\mathfrak{h}^{3}$ with a basis $X, Y, Z$ satisfying

$$
[Z, X]=X, \quad[Z, Y]=Y \text {. }
$$

The corresponding simply connected Lie group $H^{3}$ is the standard hyperbolic 3-space of constant sectional curvature -1

Example 3.6 (VI). The Lie algebra $\mathfrak{s o l}_{\alpha}^{3}$, where $\alpha>0$, is the Lie algebra with basis $X, Y, Z$ satisfying

$$
[Z, X]=\alpha X, \quad[Z, Y]=-Y .
$$

The corresponding simply connected Lie group is denoted by $\mathrm{Sol}_{\alpha}^{3}$. The group Sol mentioned in the introduction is actually $\mathrm{Sol}_{1}^{3}$.

Example 3.7 (VII). The Lie algebra $\mathfrak{g}_{7}(\alpha)$, where $\alpha \in \mathbb{R}$, is the the Lie algebra with basis $X, Y, Z$ satisfying

$$
[Z, X]=\alpha X-Y, \quad[Z, Y]=X+\alpha Y \text {. }
$$

The corresponding simply connected Lie group is denoted by $G_{7}(\alpha)$.

Example 3.8 (VIII). The Lie algebra $\mathfrak{s l}_{2}(\mathbb{R})$ with a basis $X, Y, Z$ satisfying

$$
[X, Y]=-2 Z, \quad[Z, X]=2 Y, \quad[Y, Z]=2 X .
$$

The corresponding simply connected Lie group is denoted by $\widetilde{\mathbf{S L}_{2}(\mathbb{R})}$ as it is the universal cover of the special linear group $\mathbf{S L}_{2}(\mathbb{R})$. 
Example 3.9 (IX). The Lie algebra $\mathfrak{s u ( 2 )}$ with a basis $X, Y, Z$ satisfying

$$
[X, Y]=2 Z, \quad[Z, X]=2 Y, \quad[Y, Z]=-2 X .
$$

The corresponding simply connected Lie group is of course $\mathbf{S U}(2)$. This is isometric to the standard 3 -sphere of constant curvature +1 .

\section{The ClASSIFICATION}

In this section the following theorem of Baird and Wood is applied to get a complete classification of those 3-dimensional Riemannian Lie groups which admitting harmonic morphisms to surfaces.

Theorem 4.1. [3] Let $M$ be a 3-dimensional Riemannian manifold with non-constant sectional curvature. Then there are at most two distinct conformal foliations by geodesics of $M$. If there is an open subset on which the Ricci tensor has precisely two distinct eigenvalues, then there is at most one conformal foliation by geodesics of $M$.

The simply connected 3-dimensional Riemannian Lie groups of constant sectional curvature are the standard $\mathbb{R}^{3}, H^{3}$ and $\mathbf{S U}(2)$, modulo a constant multiple of the metric. For the other cases we have the following result.

Theorem 4.2. [6] Let $G$ be a connected 3-dimensional Lie group with a left-invariant metric of non-constant sectional curvature. Then any local conformal foliation by geodesics of a connected open subset of $G$ can be extended to a global conformal foliation by geodesics of $G$. This is given by the left-translation of a 1-parameter subgroup of $G$.

Proof. Assume that $\mathcal{V}$ is a conformal foliation by geodesics of some connected neighbourhood $U$ of the identity element $e$ of $G$ and denote by $\mathcal{H}$ the orthogonal complement of $\mathcal{V}$. Let $U^{\prime} \subset U$ be a connected neighbourhood of $e$ such that $g h \in U$ for all $g, h \in U^{\prime}$, and let $U^{\prime \prime} \subset U^{\prime}$ be a connected neigbourhood of $e$ for which $g^{-1} \in U^{\prime}$ for all $g \in U^{\prime \prime}$.

For any $g \in G$, we denote by $L_{g}: G \rightarrow G$ left translation by $G$. Take $g \in U^{\prime \prime}$ and consider the distribution $\left.d L_{g} \mathcal{V}\right|_{U^{\prime}}$, obtained by restricting $\mathcal{V}$ to $U^{\prime}$ and translating with $g$. As $L_{g}$ is an isometry, this is also a conformal foliation by geodesics of $L_{g} U^{\prime}$, which is a connected neigbourhood of $e$. It is clear from Theorem 4.1 and by continuity, that this distribution must coincide with $\mathcal{V}$ restricted to $L_{g} U^{\prime}$. It follows that $d\left(L_{g}\right)_{h}\left(\mathcal{V}_{h}\right)=\mathcal{V}_{g h}$ for all $g, h \in U^{\prime \prime}$. In particular we have

$$
d\left(L_{g}\right)_{e}\left(\mathcal{V}_{e}\right)=\mathcal{V}_{g} \quad\left(g \in U^{\prime \prime}\right) .
$$

Define a 1-dimensional distribution $\tilde{\mathcal{V}}$ on $G$ by

$$
\tilde{\mathcal{V}}_{g}=\left(d L_{g}\right)_{e}\left(\mathcal{V}_{e}\right) \quad(g \in G) .
$$

Its horizontal distribution $\tilde{\mathcal{H}}$ is clearly given by left translation of $\mathcal{H}_{e}$. From the above we see that

$$
\left.\tilde{\mathcal{V}}\right|_{U^{\prime \prime}}=\left.\mathcal{V}\right|_{U^{\prime \prime}}
$$


It follows that

$$
\left.B^{\tilde{\mathcal{V}}}\right|_{U^{\prime \prime}}=\left.B^{\mathcal{V}}\right|_{U^{\prime \prime}}=0
$$

and since $\tilde{\mathcal{V}}$ is left-invariant, it follows that $B^{\tilde{\mathcal{V}}}=0$ everywhere, i.e., $\tilde{\mathcal{V}}$ is totally geodesic. In the same way we see that $\tilde{\mathcal{V}}$ is a conformal distribution and, by Theorem 4.1, we see that

$$
\left.\tilde{\mathcal{V}}\right|_{U}=\mathcal{V}
$$

This shows that $\mathcal{V}$ extends to a global conformal, totally geodesic distribution $\tilde{\mathcal{V}}$, which is left-invariant. By picking any unit vector $V \in \mathcal{V}_{e}$, we see that the corresponding foliation is given by left translation of the 1-parameter subgroup generated by $V$.

Let $G$ be a 3 -dimensional Lie group with Lie algebra $\mathfrak{g}$ equipped with a left-invariant Riemannian metric such that $\{X, Y, Z\}$ is an orthonormal basis for $\mathfrak{g}$. Assume that the 1-dimensional left-invariant foliation generated by $Z \in \mathfrak{g}$ is minimal and horizontally conformal i.e. producing harmonic morphisms. Then it is easily seen that the bracket relations for $\mathfrak{g}$ are of the form

$$
\begin{aligned}
& {[X, Y]=x X+y Y+z Z,} \\
& {[Z, X]=a X+b Y,} \\
& {[Z, Y]=-b X+a Y,}
\end{aligned}
$$

where $a, b, x, y, z \in \mathbb{R}$. In this case the Jacobi identities for the Lie algebra $\mathfrak{g}$ imply that

$$
a z=0, \quad a x+b y=0, \quad b x-a y=0 .
$$

The following three families of 3-dimensional Lie algebras give a complete classification.

Example 4.3. With $a=b=0$ we yield a 3-dimensional family of Lie groups with bracket relation

$$
[X, Y]=x X+y Y+z Z .
$$

If $x=y=z=0$ then the type is I. If $x$ or $y$ non-zero then we have type III. If $z \neq 0$ and $x=y=0$, then the type is II.

Example 4.4. In the case of $x=y=z=0$ we get semi-direct products $\mathbb{R}^{2} \rtimes \mathbb{R}$ with bracket relations

$$
\begin{aligned}
& {[Z, X]=a X+b Y,} \\
& {[Z, Y]=-b X+a Y .}
\end{aligned}
$$

If $b \neq 0$ then the Lie algebra is of type VII. If $b=0$ then the Lie algebra is of type $\mathrm{V}$ or of type I if also $a=0$.

Example 4.5. When $x=y=a=0$ we obtain a 2-dimensional family with the bracket relations

$$
[X, Y]=z Z
$$




$$
\begin{aligned}
& {[Z, X]=b Y,} \\
& {[Z, Y]=-b X .}
\end{aligned}
$$

When $b z<0$ the Lie algebra is of type VIII and of type IX if $b z>0$. The case when $z=0$ and $b \neq 0$ is of type VII $(\alpha=0)$, and the case when $b=0$ and $z \neq 0$ is of type II. The case when $b=z=0$ is of type I.

With the above analysis we have proved the following classification result.

Theorem 4.6. Let $G$ be a 3 -dimensional Lie group with Lie algebra $\mathfrak{g}$. Then there exists a left-invariant Riemannian metric $g$ on $G$ and a left-invariant horizontally conformal foliation on $(G, g)$ by geodesics if and only if the Lie algebra $\mathfrak{g}$ is neither of type IV nor of type VI.

Note that in the cases of type I, II, III, V, VII, VIII and IX the possible left-invariant Riemannian metrics are completely determined via isomorphisms to the standard examples presented in Section 3 .

\section{REFERENCES}

[1] P. Baird and J. Eells, A conservation law for harmonic maps, Geometry Symposium Utrecht 1980, Lecture Notes in Mathematics 894, 1-25, Springer (1981).

[2] P. Baird and J. C. Wood, Harmonic morphisms, Seifert fibre spaces and conformal foliations, Proc. London Math. Soc. 64 (1992), 170-197.

[3] P. Baird and J. C. Wood, Harmonic morphisms between Riemannian manifolds, London Math. Soc. Monogr. No. 29, Oxford Univ. Press (2003).

[4] B. Fuglede, Harmonic morphisms between Riemannian manifolds, Ann. Inst. Fourier 28 (1978), 107-144.

[5] S. Gudmundsson, The Bibliography of Harmonic Morphisms, http://www.matematik.lu.se/ matematiklu/personal/sigma/harmonic/bibliography.html

[6] S. Gudmundsson and M. Svensson Harmonic morphisms from solvable Lie groups, Math. Proc. Cambridge Philos. Soc. 147 (2009), 389-408.

[7] T. Ishihara, A mapping of Riemannian manifolds which preserves harmonic functions, J. Math. Soc. Japan 7 (1979), 345-370.

[8] C. G.J. Jacobi, Über eine Lösung der partiellen Differentialgleichung $\frac{\partial^{2} V}{\partial x^{2}}+\frac{\partial^{2} V}{\partial y^{2}}+\frac{\partial^{2} V}{\partial z^{2}}=0$, J. Reine Angew. Math. 36 (1848), 113-134.

[9] M. Ville, Harmonic morphisms from Einstein 4-manifolds to Riemann surfaces, Internat. J. Math. 14 (2003), 327-337.

Department of Mathematics, Faculty of Science, Lund University, Box 118, S-221 00 LUND, SWEDEN

E-mail address: Sigmundur.Gudmundsson@math.lu.se

Department of Mathematics \& Computer Science, and $\mathrm{CP}^{3}$-Origins Centre of ExCellence for Particle Physics Phenomenology, University of Southern Denmark, CamPusvej 55, DK-5230 Odense M, Denmark

E-mail address: svensson@imada.sdu.dk 